\title{
Takagi-Sugeno Fuzzy Observer and Extended-Kalman Filter for Adaptive Payload Estimation
}

\author{
Selami Beyhan, Zsófia Lendek, Musa Alcı, Robert Babuška
}

\begin{abstract}
In this paper, two nonlinear state estimation methods, Takagi-Sugeno fuzzy observer and extended-Kalman filter are compared in terms of their ability to reliably estimate the velocity and an unknown, variable payload of a nonlinear servo system. Using the system dynamics and a position measurement, the velocity and unknown payload are estimated. In a simulation study, the servo system is excited with a randomly generated step input. In real-time experiments, the estimation is performed under feedback-linearizing control. The performance of the TS fuzzy payload estimator is discussed with respect to the choice of the desired convergence rate. The application results show that the Takagi-Sugeno fuzzy observer provides better performance than the extended-Kalman filter with robust and less parameter dependent structure.
\end{abstract}

\section{INTRODUCTION}

The knowledge of the evolution of the states of nonlinear systems is essential for monitoring, feedback control and decision making. However, in general, some states cannot be measured due the lack of sensors. State observers have first been introduced for linear systems [1], and later developed for nonlinear systems [2]. To design an observer for a nonlinear system, the system has to be described in a statespace form. Takagi-Sugeno (TS) fuzzy systems [3] provide a generic nonlinear state-space model that can approximate any nonlinear system on a compact set with an arbitrary accuracy. A given nonlinear system can be represented or approximated by TS fuzzy systems using dynamic linearization [4], sector nonlinearity approach [5] or substitution [6]. Stability and by an extension, observer design conditions for TS fuzzy systems, rely on linear matrix inequalities (LMIs) [7], [8]. Some of the observers used to estimate the states of TS fuzzy systems are fuzzy Thau-Luenberger observers [9], [10], [11], sliding mode observers [12], [13] and adaptive fuzzy observer [14]. By using the observers, the unmeasurable states can be estimated, analyzed and used for the control of nonlinear systems.

Several methods have been developed in the literature to estimate the unknown payload of robot manipulators and other mechatronic systems. Off-line trained fuzzy or neural

Selami Beyhan, Department of Electrical and Electronics Engineering, Pamukkale University, Kınıklı Campus, 20040 Denizli, Turkey, sbeyhan@pau.edu.tr

Zsófia Lendek, Department of Automation, Technical University of Cluj-Napoca, Memorandumului nr. 28, 400114 Cluj-Napoca, Romania, zsofia.lendek@aut.utcluj.ro

Musa Alc1, Department of Electrical and Electronics Engineering, Ege University, Bornova, Izmir, 35100, Turkey, musa.alci@ege.edu.tr

Robert Babuška, Delft Center for Systems and Control, Delft University of Technology, Mekelweg 2, 2628 CD Delft, The Netherlands, r.babuska@tudelft.nl systems have been designed in [15], [16]. However, these payload estimators have a large number of parameters, which are trained using the recorded position, velocity and known payload information. In order to implement these approaches in a real time application, the correct position and velocity information is needed, which cannot be measured for all systems. In [17], an adaptive system based on the prediction error minimization is proposed that estimates the correct payload online, but again the measured velocity is needed. In [18], a neural network model is trained off-line with a large data-set and a Kalman filter is used to online estimation of the payload. The estimated payload has been used in [15], [16] to improve the control performance of the manipulator.

In the present paper, an estimator of an unknown and variable payload in a nonlinear servo system has been developed by using extended-Kalman filter (EKF) and Takagi-Sugeno fuzzy observer (TSFO).

The rest of the paper is organized as follows. The TakagiSugeno fuzzy modeling and observer design are explained in Section II. A brief introduction of the extended-Kalman filter is given in Section III. To estimate the payload, the specific TS fuzzy observer and EKF designs are given in Sections IV-A and IV-B respectively. Section V presents simulation results for random input excitation. Real-time estimation results are given in Section VI for the situation when the servo system is controlled by using feedbacklinearizing control. Finally, Section VII concludes the paper.

\section{TAKAGI-SUGENO FUZZY OBSERVER DESIGN}

\section{A. TS fuzzy system modeling}

In order to design a TS fuzzy observer, the nonlinear system under study is first represented as a TS fuzzy model. One possibility to construct the TS model is by using the sector nonlinearity approach [5]. This approach gives the TS model as an exact representation of an input-affine nonlinear system of the form

$$
\begin{aligned}
& \dot{\mathbf{x}}=\mathbf{A}(\mathbf{x}) \mathbf{x}+\mathbf{B}(\mathbf{x}) \mathbf{u}+\mathbf{a}(\mathbf{x}), \\
& \mathbf{y}=\mathbf{C}(\mathbf{x}) \mathbf{x}+\mathbf{c}(\mathbf{x}),
\end{aligned}
$$

defined on a compact set. The TS fuzzy model has the following form

$$
\begin{aligned}
\dot{\mathbf{x}} & =\sum_{i=1}^{M} h_{i}(\mathbf{z})\left(\mathbf{A}_{i} \mathbf{x}+\mathbf{B}_{i} \mathbf{u}+\mathbf{a}_{i}\right), \\
\mathbf{y} & =\sum_{i=1}^{M} h_{i}(\mathbf{z})\left(\mathbf{C}_{i} \mathbf{x}+\mathbf{c}_{i}\right),
\end{aligned}
$$


where $\mathbf{x}$ is the state vector, $\mathbf{y}$ is the output vector, $\mathbf{u}$ is the known input vector, $\mathbf{A}_{i}, \mathbf{B}_{i}$ and $\mathbf{C}_{i}$ are the local matrices, and $\mathbf{a}_{i}$ and $\mathbf{c}_{i}$ are the local affine terms. The scheduling vector $\mathbf{z}$ is constructed using the nonlinear terms in (1). The nonlinear terms in (1) are defined as the scheduling variable $z_{j}(\cdot) \in\left[\underline{n l}_{j}, \overline{n l}_{j}\right], j=1,2, \ldots, p$, where $\underline{n l}_{j}$ and $\overline{n l}_{j}$ are the minimum and maximum values of the $j$ th scheduling variable's domain. Using the $\underline{n l}_{j}$ and $\overline{n l}_{j}$ values, two weighting functions are defined for each nonlinearity as

$$
w_{0}^{j}(\cdot)=\frac{\overline{n l}_{j}-z_{j}(\cdot)}{\underline{n l}_{j}-\overline{n l}_{j}} \quad w_{1}^{j}(\cdot)=1-w_{0}^{j}(\cdot) \quad j=1,2, \ldots, p .
$$

Each scheduling variable $z_{j}(\cdot), j=1,2, \ldots, p$ is then written as the weighted sum of the minimum and maximum values as $z_{j}(\cdot)=\underline{n l}_{j} w_{0}^{j}(\cdot)+\overline{n l}_{j} w_{1}^{j}(\cdot)$, where $w_{0}^{j}(\cdot) \geq 0, w_{1}^{j}(\cdot) \geq 0$ and $w_{0}^{j}(\cdot)+w_{1}^{j}(\cdot)=1$.

In general, using $p$ scheduling variables, the fuzzy rule base is written as:

Rule i:

$$
\begin{aligned}
& \text { if } z_{1} \text { is } Z_{1}^{i} \text { and ... and } z_{p} \text { is } Z_{p}^{i} \text { then } \\
& \qquad \begin{aligned}
\dot{\mathbf{x}} & =\mathbf{A}_{i} \mathbf{x}+\mathbf{B}_{i} \mathbf{u}+\mathbf{a}_{i} \\
\mathbf{y} & =\mathbf{C}_{i} \mathbf{x}+\mathbf{c}_{i}
\end{aligned}
\end{aligned}
$$

where $Z_{j}^{i}, i=1,2, \ldots, m, j=1,2, \ldots, p$ are given by the weighting functions. Therefore, the TS fuzzy system has $M=2^{p}$ rules. The $i$ th membership function of the TS fuzzy system is calculated by the product of the weighting functions as

$$
h_{i}(\mathbf{z})=\prod_{j=1}^{p} w_{i j}\left(z_{j}\right)
$$

where $w_{i j}(z)$ represents the $i$ th rule $j$ th scheduling variable weighting function, that can be one of the values of $w_{0}^{j}(\cdot)$ and $w_{1}^{j}(\cdot)$ depending on which one is used in the corresponding rule. The $\mathbf{A}_{i}, \mathbf{B}_{i}, \mathbf{C}_{i}, \mathbf{a}_{i}, \mathbf{c}_{i}$ terms in (2) are constructed by using the corresponding combinations of the maximum and minimum values of the nonlinearities.

\section{B. TS fuzzy observer design}

The conditions for observer design for a given TS fuzzy model are in general extensions of stability conditions for autonomous TS fuzzy models. For an autonomous TS fuzzy system, conditions for the stability of the origin are given in Theorem 1.

Theorem 1: [7] The equilibrium $\mathbf{x}=0$ of the fuzzy system $\dot{\mathbf{x}}=\sum_{i=1}^{M} h_{i}(\mathbf{z}) \mathbf{A}_{i} \mathbf{x}$ is asymptotically stable, if there exists $P=P^{T}>0$ such that

$$
\mathbf{A}_{i}^{T} \mathbf{P}+\mathbf{P} \mathbf{A}_{i}<0
$$

for $i=1,2, \ldots, M$.

The existence of the common $\mathbf{P}$ matrix can be tested by solving linear matrix inequalities [9].

The so-called "Thau-Luenberger fuzzy observer" [10] has been frequently used for the state estimation of nonlinear systems [13], [19], [20]. In general, the scheduling vector $\mathbf{z}$ may be composed of the system's states, inputs, or measurements. In this research, we assume that $\mathbf{z}$ is measured. Then, the TS fuzzy observer has the form

$$
\begin{aligned}
\dot{\hat{\mathbf{x}}} & =\sum_{i=1}^{M} h_{i}(\mathbf{z})\left(\mathbf{A}_{i} \hat{\mathbf{x}}+\mathbf{B}_{i} u+\mathbf{a}_{i}+L_{i}(\mathbf{y}-\hat{\mathbf{y}})\right), \\
\hat{\mathbf{y}} & =\sum_{i=1}^{M} h_{i}(\mathbf{z})\left(\mathbf{C}_{i} \hat{\mathbf{x}}+\mathbf{c}_{i}\right) .
\end{aligned}
$$

where the $\mathbf{L}_{i}, i=1,2, \ldots, M$, are the observer gains. The pairs $\left(\mathbf{A}_{i}, \mathbf{C}_{i}\right)$ are assumed to be observable. The aim of the observer is to estimate the states of (2), which is achieved if the error dynamics $\dot{\hat{\mathbf{e}}}=\dot{\mathbf{x}}-\dot{\hat{\mathbf{x}}}$ are asymptotically stable. The error dynamics can be written as

$$
\dot{\hat{\mathbf{e}}}=\sum_{i=1}^{M} \sum_{j=1}^{M} h_{i}(\mathbf{z}) h_{j}(\mathbf{z})\left(\mathbf{A}_{i}-\mathbf{L}_{i} \mathbf{C}_{j}\right) \hat{\mathbf{e}} .
$$

The stability conditions are given in Theorem 2 .

Theorem 2: [7] The error dynamics (8) are asymptotically stable, if there exist $\mathbf{P}=\mathbf{P}^{T}>0, L_{i}, i=1,2, \ldots, M$, such that

$$
\begin{array}{r}
\mathcal{H}\left(\mathbf{P}\left(\mathbf{A}_{i}-\mathbf{L}_{i} \mathbf{C}_{i}\right)\right)<0, \\
\mathcal{H}\left(\mathbf{G}_{i j}+\mathbf{G}_{j i}\right) \leq 0, \\
\mathbf{G}_{i j}=\mathbf{P}\left(\mathbf{A}_{i}-\mathbf{L}_{i} \mathbf{C}_{j}\right),
\end{array}
$$

for all pairs of $(i, j), i=1,2, \ldots, M, j=1,2, \ldots, M$, for which there exists $\mathbf{z}$ such that $h_{i}(\mathbf{z}) h_{j}(\mathbf{z}) \neq 0$. The symbol $\mathcal{H}$ denotes the Hermitian defined as $\mathcal{H}(\mathbf{A})=\mathbf{A}+\mathbf{A}^{T}$.

The error dynamics of the TS observer (8) can be designed with a desired convergence rate $\alpha>0$ using Theorem 3 .

Theorem 3: [7] The convergence rate of the error dynamics (8) is at least $\alpha$, if there exist $\mathbf{P}=\mathbf{P}^{T}>0, L_{i}$, $i=1,2, \ldots, M$, such that

$$
\begin{gathered}
\mathcal{H}\left(\mathbf{P}\left(\mathbf{A}_{i}-\mathbf{L}_{i} \mathbf{C}_{i}\right)\right)+2 \alpha \mathbf{P}<0, \\
\mathcal{H}\left(\mathbf{G}_{i j}+\mathbf{G}_{j i}\right)+4 \alpha \mathbf{P} \leq 0, \\
\mathbf{G}_{i j}=\mathbf{P}\left(\mathbf{A}_{i}-\mathbf{L}_{i} \mathbf{C}_{j}\right),
\end{gathered}
$$

for all pairs of $(i, j), i=1,2, \ldots, M, j=1,2, \ldots, M$, for which there exists $\mathbf{z}$ such that $h_{i}(\mathbf{z}) h_{j}(\mathbf{z}) \neq 0$.

\section{EXTENDED-KALMAN FILTER}

The Kalman filter has been introduced as an optimal filter for linear systems in [21] and used to estimate the unmeasurable states of the linear systems for a half century [22]. For the state estimation of nonlinear systems, the Kalman filter estimate is based on the linearized system, and the filter is called "extended-Kalman filter" [23]. The Kalman filter has been also utilized for the parameter estimation in [24]. The continuous-time extended-Kalman filter dynamics are summarized as follows [22].

1. The nonlinear system dynamics are

$$
\begin{aligned}
\dot{\mathbf{x}} & =\mathbf{f}(\mathbf{x}, \mathbf{u}, w), \\
\mathbf{y} & =\mathbf{h}(\mathbf{x}, v), \\
w & \sim \mathcal{N}(0, \mathbf{Q}), \\
v & \sim \mathcal{N}(0, \mathbf{R}),
\end{aligned}
$$


where $f($.$) and g($.$) are nonlinear functions, \mathbf{x}$ is the system state and $\mathbf{u}$ is the input. In (11), $v$ and $w$ are normally distributed state and measurement noises, respectively, with $\mathbf{Q}$ and $\mathbf{R}$ the corresponding covariance matrices.

2. The linearized dynamics around the current estimate are

$$
\begin{aligned}
\mathbf{F} & =\left.\frac{\partial \mathbf{f}(\mathbf{x})}{\partial \mathbf{x}}\right|_{\mathbf{x}=\hat{\mathbf{x}}}, \\
\mathbf{N} & =\left.\frac{\partial \mathbf{f}(\mathbf{x})}{\partial w}\right|_{\mathbf{x}=\hat{\mathbf{x}}}, \\
\mathbf{H} & =\left.\frac{\partial \mathbf{h}(\mathbf{x})}{\partial \mathbf{x}}\right|_{\mathbf{x}=\hat{\mathbf{x}}}, \\
\mathbf{M} & =\left.\frac{\partial \mathbf{h}(\mathbf{x})}{\partial v}\right|_{\mathbf{x}=\hat{\mathbf{x}}} .
\end{aligned}
$$

4. The extended-Kalman filter update equations are

$$
\begin{aligned}
\dot{\hat{\mathbf{x}}} & =\mathbf{f}(\hat{\mathbf{x}}, \mathbf{u}, w)+\mathbf{K}[\mathbf{y}-\mathbf{h}(\hat{\mathbf{x}}, v)], \\
\mathbf{K} & =\boldsymbol{\Gamma} \mathbf{H}^{T} \mathbf{R}_{c}^{-1}, \\
\dot{\boldsymbol{\Gamma}} & =\mathbf{F} \boldsymbol{\Gamma}+\boldsymbol{\Gamma} \mathbf{F}^{T}+\mathbf{Q}_{c}-\boldsymbol{\Gamma} \mathbf{H}^{T} \mathbf{R}_{c}^{-1} \mathbf{H} \boldsymbol{\Gamma},
\end{aligned}
$$

where $\mathbf{K}$ is the Kalman gain matrix and $\boldsymbol{\Gamma}$ is the error covariance matrix. The initialization of $\Gamma$ is based on the estimated initial states of the system. In (14b) and (14c), the $\mathbf{K}$ and $\boldsymbol{\Gamma}$ matrices are updated using the linearized system dynamics (12) and (13). Afterward, the states of the Kalman filter (14a) are updated using $\mathbf{K}$ and error $\hat{\mathbf{e}}=y-\mathbf{h}(\hat{\mathbf{x}}, v)$. In the next section, first the nonlinear servo system dynamics are given and then TSFO and EKF designs for the servo system are explained in detail.

\section{NONLINEAR SERVO SYSTEM}

Consider a DC motor whose shaft directly drives a disk rotating in a vertical plane. A payload with an unknown mass is attached to the disk at an off-the-center position, see Figure 1.

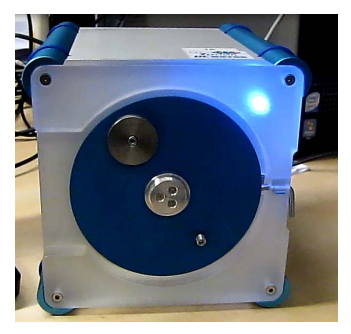

Fig. 1. A laboratory setup of the nonlinear servo system.

Due to gravity, the weight makes the dynamics nonlinear, exactly in the same way as in a 1-DOF robotic arm. The nonlinear dynamics are given by

$$
\begin{aligned}
& \dot{x}_{1}=x_{2}, \\
& \dot{x}_{2}=\frac{-K_{m}^{2}-b R_{m}}{R_{m} J} x_{2}-\frac{g L_{m}}{J} \sin \left(x_{1}\right) x_{3}+\frac{K_{m}}{R_{m} J} u, \\
& \dot{x}_{3}=0
\end{aligned}
$$

where $x_{1}$ is the angular position of the payload measured in radians from the vertical and $x_{2}$ is the angular velocity of the payload in $\mathrm{rad} / \mathrm{sec}$. The payload $m_{L}$ is unknown, but constant, and it is therefore represented as the third state of the system, $x_{3}$. The derivative $\dot{x}_{3}=0$, due to the piecewise constant nature of the payload mass. The parameters are listed in Table I.

TABLE I

PARAMETERS OF THE SERVO SYSTEM

\begin{tabular}{lll}
\hline$K_{m}$ & Electromotive force constant & $0.0536 \mathrm{~N} \mathrm{~m} / \mathrm{A}$ \\
$\mathrm{b}$ & Damping coefficient (viscous friction) & $3 \times 10^{-6} \mathrm{~kg} / \mathrm{s}$ \\
$R_{m}$ & Electric resistance & 9.5 \\
$\mathrm{~J}$ & Moment of inertia of rotor and disk & $1.91 \times 10^{-4} \mathrm{~kg} \mathrm{~m}^{2}$ \\
$\mathrm{~g}$ & Acceleration due to gravity & $9.81 \mathrm{~m} / \mathrm{s}^{2}$ \\
$L_{m}$ & Payload distance from the center & $0.042 \mathrm{~m}$ \\
$m_{L}$ & Payload & $0-150 \mathrm{gr}$ \\
\hline
\end{tabular}

\section{A. TS fuzzy observer design for servo system}

The system is represented by a TS fuzzy system of the form (2) using the sector nonlinearity approach. The servo system can be written using the matrices

$$
\begin{gathered}
\mathbf{A}=\left[\begin{array}{ccc}
0 & 1 & 0 \\
0 & \frac{-K_{m}^{2}-b R_{m}}{R_{m} J} & -\frac{g L_{m}}{J} \sin \left(x_{1}\right) \\
0 & 0 & 0
\end{array}\right], \\
\mathbf{B}=\left[\begin{array}{c}
0 \\
\frac{K_{m}}{R_{m} J} \\
0
\end{array}\right], \mathbf{a}=\left[\begin{array}{l}
0 \\
0 \\
0
\end{array}\right], \quad \mathbf{C}=\left[\begin{array}{l}
1 \\
0 \\
0
\end{array}\right]^{T} .
\end{gathered}
$$

Given the nonlinearity in the system matrix (16), the scheduling variable is selected as $z=-\frac{g L_{m}}{J} \sin \left(x_{1}\right)$ which depends on the position of the payload. To determine the local models of the TS fuzzy system, the minimum and maximum values of the position are set to $x_{1} \in[0, \pi]$ radians, in accordance with the desired positions of the payload. The state matrices of local models in the TS system are

$$
\mathbf{A}_{1}=\left[\begin{array}{ccc}
0 & 1 & 0 \\
0 & -1.6 & -2.1 \cdot 10^{3} \\
0 & 0 & 0
\end{array}\right], \mathbf{A}_{2}=\left[\begin{array}{ccc}
0 & 1 & 0 \\
0 & -1.6 & -21.6 \\
0 & 0 & 0
\end{array}\right]
$$

and the weighting functions are obtained as follows:

$$
w_{1}(z)=\frac{-2.1 \cdot 10^{3}+\frac{g L_{m}}{J} \sin \left(x_{1}\right)}{-2.1 \cdot 10^{3}+21.6}, \quad w_{2}(z)=1-w_{1}(z) .
$$

As there is one scheduling variable, two subsystems and two rules, the membership functions $h_{i}(\mathbf{z})$ are equal to the weighting functions, $w_{i}(\mathbf{z})$. The final TS fuzzy system is obtained as

$$
\begin{aligned}
\dot{\mathbf{x}} & =\sum_{i=1}^{2} w_{i}(\mathbf{z})\left(\mathbf{A}_{i} \mathbf{x}+\mathbf{B u}\right), \\
\mathbf{y} & =\mathbf{C} \mathbf{x},
\end{aligned}
$$

where $w_{i}, \mathbf{A}_{i}$, a and $\mathbf{C}$ values are defined in (18), (17) and (16), respectively. The $K_{m}, L_{m}$ and $J$ parameters are given in Table I.

The TS fuzzy system (19) representing the nonlinear servo 
system, is used to design TS fuzzy observer for velocity and payload estimation. Using Theorem 3 and $\alpha=1.5$, the observer gains are calculated as

$$
\mathbf{L}_{1}=\left[\begin{array}{c}
0.68 \\
333.33 \\
-39.97
\end{array}\right] \times 10^{3}, \quad \mathbf{L}_{2}=\left[\begin{array}{c}
18.61 \\
912.42 \\
-139.38
\end{array}\right] \times 10^{3} .
$$

To observe the effect of the $\alpha$ (decay rate) gains, different designs are compared in the next section.

\section{B. Extended-Kalman Filter Design for Servo System}

In the EKF design, the unknown payload is considered again as an external parameter and its change with respect to time is $\dot{x}_{3}=0$. We add normally distributed artificial noises $w$ and $v$ to the third state and output measurement with the variances $Q=10^{-1}$ and $R=10^{-3}$, respectively. The Kalman filter dynamics are given by

$$
\begin{aligned}
\dot{\hat{\mathbf{x}}} & =\left[\begin{array}{c}
\hat{x}_{2} \\
\frac{-K_{m}^{2}-b R_{m}}{R_{m} J} \hat{x}_{2}-\frac{g L_{m}}{J} \sin \left(\hat{x}_{1}\right) \hat{x}_{3}+\frac{K_{m}}{R_{m} J} u \\
w
\end{array}\right], \\
& =\mathbf{f}(\hat{\mathbf{x}}, u, w), \\
y & =\left[\begin{array}{lll}
1 & 0 & 0
\end{array}\right] \hat{\mathbf{x}}+v .
\end{aligned}
$$

The EKF states are updated using (14a). To calculate $\mathbf{K}$ and $\boldsymbol{\Gamma}$, the following matrices are used

$$
\begin{aligned}
\mathbf{F} & =\left.\frac{\partial \mathbf{f}(\mathbf{x})}{\partial \mathbf{x}}\right|_{\mathbf{x}=\hat{\mathbf{x}}} \\
& =\left[\begin{array}{ccc}
0 & 1 & 0 \\
-\frac{g L_{m}}{J} \cos \left(\hat{x}_{1}\right) \hat{x}_{3} & \frac{-K_{m}^{2}-b R_{m}}{R_{m} J} & -\frac{g L_{m}}{J} \sin \left(\hat{x}_{1}\right) \\
0 & 0
\end{array}\right], \\
\mathbf{H} & =\left.\frac{\partial \mathbf{h}(\mathbf{x})}{\partial \mathbf{x}}\right|_{\mathbf{x}=\hat{\mathbf{x}}}=\left[\begin{array}{lll}
1 & 0 & 0
\end{array}\right], \\
\mathbf{N} & =\left.\frac{\partial \mathbf{f}(\mathbf{x})}{\partial w}\right|_{\mathbf{x}=\hat{\mathbf{x}}}=\left[\begin{array}{l}
0 \\
0 \\
1
\end{array}\right], \\
\mathbf{M} & =\left.\frac{\partial \mathbf{h}(\mathbf{x})}{\partial v}\right|_{\mathbf{x}=\hat{\mathbf{x}}}=\left[\begin{array}{lll}
1
\end{array}\right], \\
\mathbf{Q}_{c} & =\mathbf{N Q N ^ { T }}=\left[\begin{array}{lll}
0 & 0 & 0 \\
0 & 0 & 0 \\
0 & 0 & 0.1
\end{array}\right], \\
\mathbf{R}_{c} & =\mathbf{M R M}^{T}=\left[\begin{array}{ll}
R & =10^{-3} .
\end{array}\right.
\end{aligned}
$$

In what follows, the observer design methods of Sections IVA and IV-B are applied to the servo system in simulation and in real-time experiments.

\section{Simulation Results}

The goal is to estimate the velocity and the mass of the payload using the model of the system and the position measurement. A random piecewise constant input signal (Figure 2) is used, designed so that the position of the payload remains inside the range of observer design specification.

The simulation lasts for $12 \mathrm{~s}$ and within this interval, the input signal is changed as $[2,5,3,4]$ volts and the payload

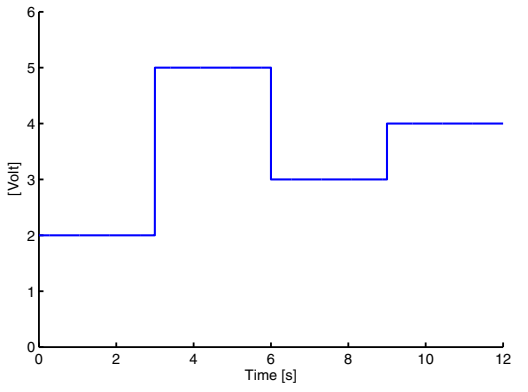

Fig. 2. Input signal.

varies as $[100,150,90]$ grams, respectively. The observers are designed in the continuous-time domain and then discretized with the sampling time of $2 \mathrm{~ms}$. The initial states of the EKF and TSFO are chosen as $\hat{\mathbf{x}}(0)=\left[\begin{array}{lll}0.01 & 0 & 0\end{array}\right]^{T}$.

The resulting velocity and velocity estimation errors are shown in Figures 3(a) and 3(b), respectively. The velocity estimation results are very accurate and estimated values rapidly converge to the true values.

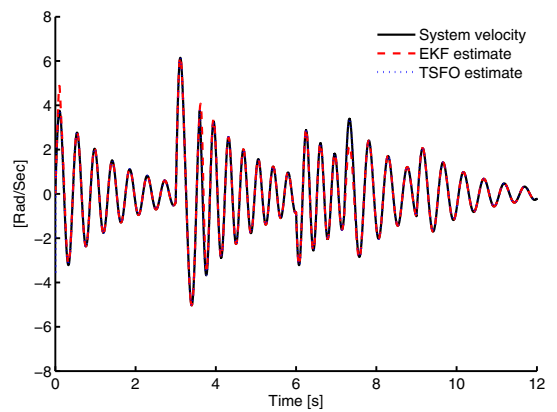

(a) Velocity estimate

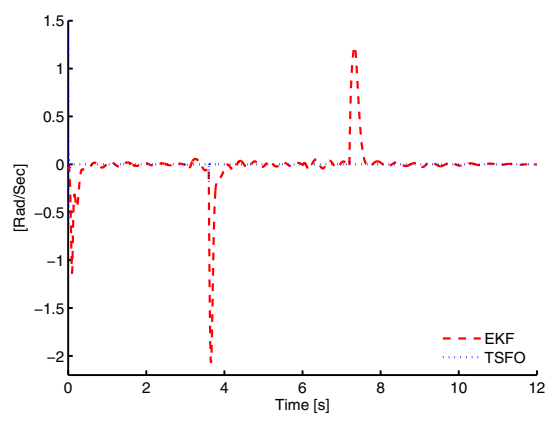

(b) Velocity estimation error

Fig. 3. Velocity estimation results (simulation).

The estimate of the payload and the payload estimation error are presented in Figures 4(a) and 4(b). Although both estimates are acceptable, TSFO payload estimation is more accurate and the convergence is faster.

Relatively large peaks in the estimation errors occur when the payload value changes. However, this estimation error converges to zero very quickly. In Figure 4(b) we observe that after the change of the payload, the TSFO estimate converges more quickly to the true value of the payload than the EKF estimate.

Table II gives the integral sum of absolute estimation 


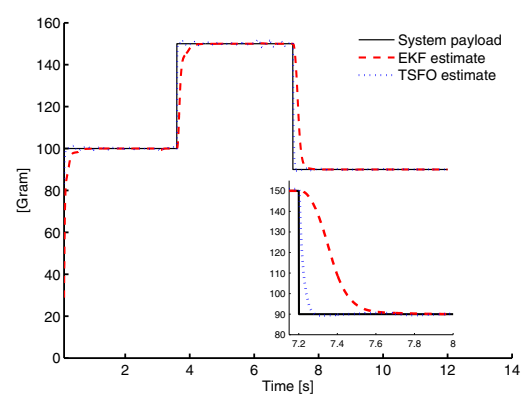

(a) Payload estimate

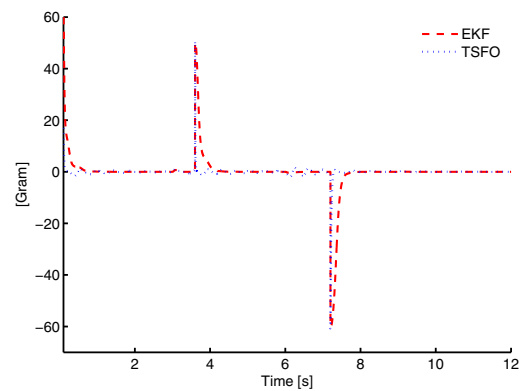

(b) Payload estimation error

Fig. 4. Payload estimation results (simulation).

TABLE II

SIMULATION PERFORMANCE

\begin{tabular}{lcc}
\hline$I A E$ & Velocity & Payload \\
\hline EKF & 0.797 & 0.031 \\
TSFO $(\alpha=10)$ & 0.021 & 0.013 \\
\hline
\end{tabular}

error $\left(I A E=\int_{0}^{T}|\hat{e}| d t\right)$ for the two observers. It must be noted that the choice of the $\alpha$ value in (9) is important for the performance of TSFO. The IAE obtained with several selected values of $\alpha$ is shown in Table III. The TSFO results in the figures are plotted using the $\alpha=10$ convergence rate.

TABLE III

PERFORMANCE OF TSFO FOR DIFFERENT $\alpha$ VALUES

\begin{tabular}{lcc}
\hline$I A E$ & Velocity & Payload \\
\hline$\alpha=0$ & 1.540 & 0.041 \\
$\alpha=5$ & 0.031 & 0.015 \\
$\alpha=10$ & 0.021 & 0.013 \\
\hline
\end{tabular}

\section{EXPERIMENTAL RESULTS}

In this part, the servo system is controlled by feedback linearization control [25], to track the reference position $x_{1}=\pi / 2$ in a real-time experiment. The changes on the reference signal (Figure 6(a)) are due to the change of the payload value. However, the controller works adequately and produces the control signal shown in Figure 5.

The EKF and TSFO observers work independently of the controller, and the estimates obtained are illustrated in Figures 6(a) and 6(b). It can be seen that the state estimates are very accurate in the real-time control setting as well. A constant 68 gram payload is mounted at one side of the disc, while smaller payloads are added to other side. In this way,

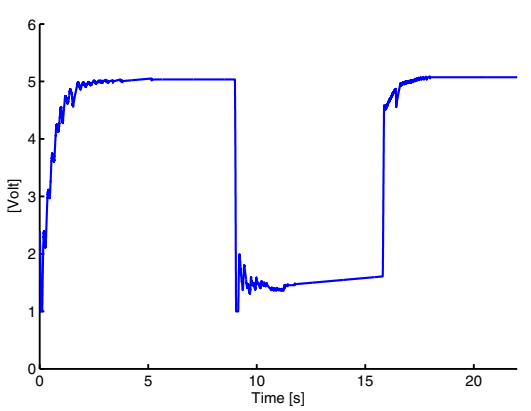

Fig. 5. Input signal.

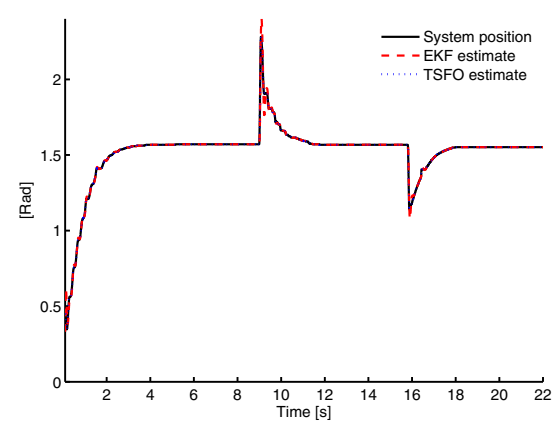

(a) Position estimate

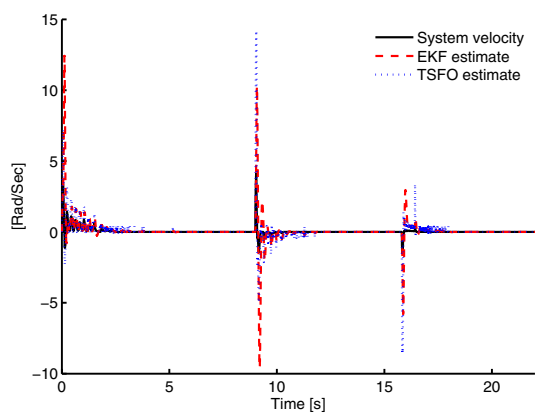

(b) Velocity estimate

Fig. 6. Estimation results (real-time).

we obtain a variable payload and are able to compare the performance of the EKF and TSFO.

The real-time performance of the two observers is compared using the $I A E$ performance measure, see Table IV. Both the numerical results in this table as well as the signals presented in Figure 7(b) show that TSFO provides a better real-time estimate of the payload and velocity.

TABLE IV

REAL-TIME PERFORMANCE

\begin{tabular}{lcc}
\hline$I A E$ & Velocity & Payload \\
\hline EKF & 4.37 & 0.054 \\
TSFO $(\alpha=1)$ & 3.14 & 0.038 \\
\hline
\end{tabular}

\section{CONCLUSION}

A Takagi-Sugeno fuzzy observer (TSFO) and an extendedKalman filter (EKF) were used to estimate the velocity and the payload of a nonlinear servo system. The TSFO is a nonlinear observer that uses the sector nonlinearity approach 


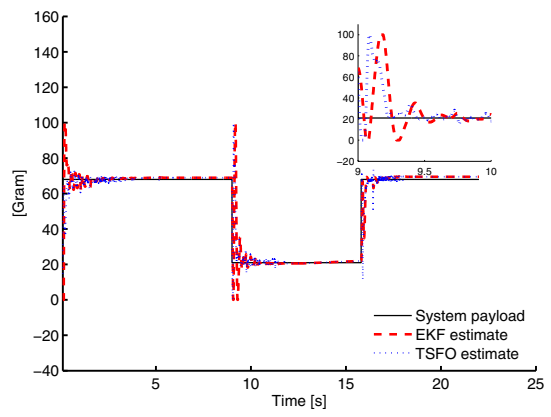

(a) Payload estimate

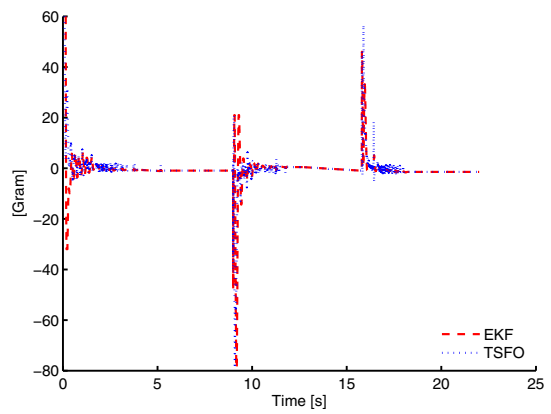

(b) Payload estimation error

Fig. 7. Payload estimation results (real-time).

to model the nonlinear system under study. The TSFO can be designed using a desired convergence rate. The EKF uses the linearized dynamics to compute the estimates. Based on our experiments, the initial values of the states and error covariance matrix affects the EKF performance. When the initialization of the states or parameter are not proper or there exist highly nonlinear dynamics, there exists divergence of the state and parameter estimations. On the other hand, the TSFO performs accurately for any value within its design region with less parameter-dependent structure.

The estimation results obtained show that the information based on the exact knowledge of the nonlinear system in TSFO design provides better state and parameter estimation results than EKF, both in simulations and in a real-time control. The ongoing works are about the adaptive payload estimation based real-time control of a flexible transmission system.

\section{ACKNOWLEDGEMENT}

This work was supported by a grant of the Romanian National Authority for Scientific Research, CNCS UEFISCDI, project number PN-II-RU-TE-2011-3-0043, contract number 74/05.10.2011, and also supported by Pamukkale University Scientific Research Projects (BAP) Department.

\section{REFERENCES}

[1] D. Luenberger, "Observers for multivariable systems," Automatic Control, IEEE Transactions on, vol. 11, no. 2, pp. 190 - 197, Apr. 1966.

[2] E. E. Thau, "Observing the state of nonlinear systems," International Journal of Control, vol. 17, pp. 471-479, 1973.

[3] T. Takagi and M. Sugeno, "Fuzzy identification of systems and its applications to modeling and control," IEEE Transactions on Systems Science and Cybernetics, vol. 15, no. 1, pp. 116-132, 1985.
[4] T. A. Johansen, R. Shorten, and R. Murray-Smith, "On the interpretation and identification of dynamic Takagi-Sugeno fuzzy models," IEEE Transactions on Fuzzy Systems, vol. 8, no. 3, pp. 297-313, 2000.

[5] H. Ohtake, K. Tanaka, and H. O. Wang, "Fuzzy modeling via sector nonlinearity concept," in IFSA World Congress and 20th NAFIPS International Conference, vol. 1, Vancouver, British Columbia, Canada, 2001, pp. 127-132.

[6] K. Kiriakidis, "Nonlinear modeling by interpolation between linear dynamics and its application in control," Journal of Dynamic Systems, Measurement, and Control, vol. 129, no. 6, pp. 813-824, 2007.

[7] K. Tanaka, T. Ikeda, and H. O. Wang, "Fuzzy regulators and fuzzy observers: relaxed stability conditions and LMI-based designs," IEEE Transactions on Fuzzy Systems, vol. 6, no. 2, pp. 250-265, 1998.

[8] P. Bergsten, R. Palm, and D. Driankov, "Fuzzy observers," in The 10th IEEE International Conference on Fuzzy Systems, vol. 2, The University of Melbourne, Australia, 2001, pp. 700-703.

[9] K. Tanaka and H. Wang, "Fuzzy regulators and fuzzy observers: a linear matrix inequality approach," in Proceedings of the 36th IEEE Conference on Decision and Control, vol. 2, San Diego, California USA, 1997, pp. $1315-1320$.

[10] R. Palm and D. Driankov, "Towards a systematic analysis of fuzzy observers," in 18th International Conference of the North American Fuzzy Information Processing Society, New York, NY, USA, 1999, pp. 179-183.

[11] P. Bergsten, R. Palm, and D. Driankov, "Observers for Takagi-Sugeno fuzzy systems," IEEE Transactions on Systems, Man, and Cybernetics, Part B: Cybernetics, vol. 32, no. 1, pp. 114-121, 2002.

[12] P. Bergsten and R. Palm, "Thau-Luenberger observers for TS fuzzy systems," in The Ninth IEEE International Conference on Fuzzy Systems, vol. 2, San Antonio, Texas, 2000, pp. 671-676.

[13] M. Oudghiri, M. Chadli, and A. E. Hajjaji, "Lateral vehicle velocity estimation using fuzzy sliding mode observer," in Mediterranean Conference on Decision and Control, Athens, Greece, 2007, pp. 1-6.

[14] J.-H. Park, P.-S. Yoon, and G.-T. Park, "Robust adaptive observer using fuzzy systems for uncertain nonlinear systems," in The 10th IEEE International Conference on Fuzzy Systems, vol. 3, The University of Melbourne, Australia, 2001, pp. 749-752.

[15] M. Leahy, M. Johnson, and S. Rogers, "Neural network payload estimation for adaptive robot control," IEEE Transactions on Neural Networks, vol. 2, no. 1, pp. 93-100, 1991.

[16] H. C. Nho and P. Meckl, "Intelligent feedforward control and payload estimation for a two-link robotic manipulator," IEEE/ASME Transactions on Mechatronics, vol. 8, no. 2, pp. 277-283, 2003.

[17] S. Abiko and K. Yoshida, "On-line parameter identification of a payload handled by flexible based manipulator," in Proceedings of the International Conference on Intelligent Robots and Systems, vol. 3, Sendai, Japan, 2004, pp. 2930-2935.

[18] M. Savia and H. N. Koivo, "Neural-network-based payload determination of a moving loader," Control Engineering Practice, vol. 12, no. 5, pp. 555-561, 2004.

[19] E. Herrera, B. Castillo, J. Ramirez, and E. C. Ferreira, "Exact fuzzy observer for a baker's yeast fed-batch fermentation process," in IEEE International Fuzzy Systems Conference FUZZ-IEEE 2007, London, England, 2007, pp. 1-6.

[20] Zs. Lendek, R. Babuška, and B. De Schutter, "Fuzzy models and observers for freeway traffic state tracking," in American Control Conference, Baltimore, MD, USA, 2010, pp. 2278-2283.

[21] R. E. Kalman, "A new approach to linear filtering and prediction problems," Transactions of the ASME-Journal of Basic Engineering, vol. 82, no. Series D, pp. 35-45, 1960.

[22] D. Simon, Optimal State Estimation: Kalman, H Infinity, and Nonlinear Approaches. Wiley-Interscience, 2006.

[23] H. Cox, "On the estimation of state variables and parameters for noisy dynamic systems," IEEE Transactions on Automatic Control, vol. 9, no. 1 , pp. $5-12$, jan 1964 .

[24] V. Aidala, "Parameter estimation via the kalman filter," Automatic Control, IEEE Transactions on, vol. 22, no. 3, pp. $471-472$, jun 1977.

[25] J. Slotine and W. Li, Applied Nonlinear Control. Prentice-Hall Inc., 1991. 